\title{
From Planetary Nebula Nuclei to White Dwarfs: The Impact of Evolutionary Envelope Masses
}

\author{
T. Blöcker ${ }^{1}$, F. Herwig ${ }^{2}$, T. Driebe ${ }^{2}$, H. Bramkamp ${ }^{2}$ and D. Schönberner ${ }^{2}$ \\ ${ }^{1}$ Institut für Astronomie und Astrophysik, 24098 Kiel, Germany; \\ ${ }^{2}$ Astrophysikalisches Institut Potsdam, 14473 Potsdam, Germany
}

It is well known that the evolution of white dwarfs (WDs) depends sensitively on the question whether they have "thin" or "thick" envelopes of H and He (see Wood 1995). Standard evolutionary caluclations (e.g. Paczynksi 1971) show that at the tip of the Asymptotic Giant Branch the envelope masses are tightly correlated with the mass of the hydrogen exhausted core ( $\approx$ total mass). Accordingly, the masses of hydrogen, $M_{\mathrm{H}}$, and helium, $M_{\mathrm{He}}$, on top of the degenerate $\mathrm{C} / \mathrm{O}$ interiors decrease by orders of magnitudes with increasing stellar mass. In contrast, many applications of WD calculations consider only single values of $q_{\mathrm{H}, \mathrm{He}}=\log \left(M_{\mathrm{H}, \mathrm{He}} / M_{*}\right)$ asuming either "thick" or "thin" envelopes.

In order to study the effects of evolutionary envelope masses on the mass-radius relation and the mass distribution of DA white dwarfs, we considered the evolutionary models of Blöcker (1995) and Driebe (1996) for C/O and He WDs, and constructed additional WD models of same mass which exhibit only purely thick or thin $\mathrm{H}$ and $\mathrm{He}$ layers.

In the case of the fully evolutionary C/O WDs the hydrogen layer varies from $10^{-4} M_{\odot}$ for $M_{\mathrm{WD}}=0.6 M_{\odot}$ down to $10^{-6} M_{\odot}$ for $M_{\mathrm{WD}}=1 M_{\odot}$. Consequently, the corresponding mass-radius relation is considerably steeper than the ones based on the assumption of constant layer thickness. White dwarfs less massive than $\approx 0.45 M_{\odot}$ are He WDs, and have been formed by binary evolution (e.g. Kippenhahn et al. 1967). Our simulation of this evolutionary channel gives hydrogen layers, which are even more massive than those of low-mass C/O WDs (up to one order of magnitude) increasing with decreasing WD mass. The mass-radius relation of He WDs is considerably steeper than the one given by C/O WDs.

Consequently, the change of the internal composition at $M_{*} \approx 0.45 M_{\odot}$ as well as the tight correlation between the H/He layers and the WD mass should be taken into account for the construction of a mass-radius relation. The resulting WD mass distribution (based on the sample of Bergeron et al. (1992)) shows no objects below $0.35 M_{\odot}$ due to the inclusion of He WDs. It gives nearly the same mean mass as one based only on thick layers. However, its shape is even more narrow since the mass-dependence of the layer thickness shifts both the low and high gravity objects towards the distribution's center.

\section{REFERENCES}

Bergeron, P., Saffer, R.A., Liebert, J.: 1992, ApJ 394, 228.

Blöcker, T.: 1995, A\&A 297, 727; A\&A 299, 755.

Driebe, T.: 1996, Diploma thesis, in prep.

Kippenhahn, R., Kohl, K., Weigert, A.: 1967, Zeitschrift f. Astrophys. 66, 58.

Paczyński, B: 1971, Acta Astron. 21, 417.

Wood, M.: 1995, in White Dwarfs, eds. D. Koester \& K. Werner, Springer, p. 41. 